
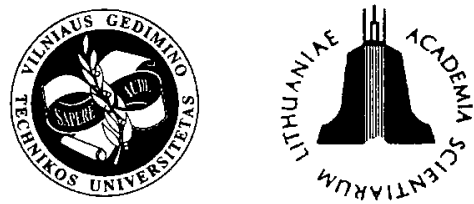

JOURNAL OF CIVIL ENGINEERING AND MANAGEMENT

http:/www.vtu. It/english/editions

2002. Vol VIII. No 4, 269-275

\title{
GRAPHO-ANALYTICAL METHOD FOR DETERMINING POSSIBILITIES OF USING DISCRETE MULTIUSE (DMU) RESOURCES IN CIVIL ENGINEERING COMPANIES
}

\author{
Romualdas Tamošaitis \\ Dept of Construction Technology and Management, Vilnius Gediminas Technical University (VGTU). \\ Sauletekio al.11,LT-2040 Vilnius,Lithuania.E-mail: roma@adm.vtu.lt
}

Received 05 June 2001; accepted 12 Sept 2002

\begin{abstract}
DMu resources in civil engineering companies include: small teams of builders, equipment pieces for performing jobs, cranes and other building machines. Limited quantities of the DMu resources often predetermine the terms of building operations and other important indices characterising the activities of civil engineering companies. This article deals with grapho-analytical method for specifying numbers of variants of distributions DMu resources and simulation of possible variants of distributions in accordance with the real job conditions.

Simulation of the variants of DMu resources of building companies has few stages. During the first stage it is necessary to determine the basic parameters of the task (the degree of providing the work programme with DMu resources, the number of $\mathrm{DMu}$ resources application variants, etc.). During the second stage, simulation of the variants of DMu resources application should be performed. The third stage should include the calculation of the economical efficiency of variants.

As an experience shows, the largest amount of computation time may be saved and the problem solved in a most reasonable way, when the task parameters are calculated accurately during the first stage and then, according to these parameters, the second stage of the task is optimised. Besides, sometimes the simulation problem cannot be solved because of the shortage of DMu resources or due to an immense number of DMu resources application variants. It may require choosing another method for task solving or changing entirely the job performance conditions.
\end{abstract}

Keywords: construction, grapho-analytical method, simulation, discrete multiuse resources.

\section{Introduction}

Civil engineering companies often encounter problems of distributing the so-called discrete resources for multiple use. These DMu resources include: small teams of builders, equipment pieces for performing jobs, cranes and other building machines. Limited quantities of the $\mathrm{DMu}$ resources often predetermine the terms of building operations and other important indices characterising the activities of building companies [1-3].

Because of limited quantities of DMu resources the problems of distributing them are solved by means of simulation $[3,4]$. It allows to assess in a more efficient way the variants of using DMu resources and to apply the multicriteria methods for evaluating those variants $[5,6]$. In order to save computation time and to simplify simulation, special programme languages are being developed for solving simulation tasks $[7,8]$.

When trying by simulation methods to find rational use variants of DMu resources, it necessary to divide the process of search in stages because the volume of calculations is rather large. During the first stage it is necessary to determine the basic parameters of the task (the degree of providing the work programme with $\mathrm{DMu}$ resources, the number of DMu resources application vari- ants, etc). During the second stage, simulation of variants of DMu resources application should be performed. The third stage should include the calculation of the economical efficiency of variants [9].

As an experience shows, the largest amount of computation time may be saved and the problem solved in a most reasonable way, when the task parameters are calculated accurately during the first stage and then, according to these parameters, the second stage of the task is optimized. Besides, sometimes the simulation problem cannot be solved because of the shortage of DMu resources or due to an immense number of DMu resources application variants. It may require choosing another method for task solving or changing entirely the job performance conditions.

\section{Simulation of the DMu resources application vari- ants}

If variants of the DMu resources application do not influence the succession of processes, we are able to simplify the problem of simulation of the DMu resources application variants. 
Let's group the technological processes according to the planned start of work and number them in succession. In this way, we can describe the resource application variants by the set $U$ in the sequence according to the work start. The state of the system to be simulated can be described by matrix $Z$, which is to fix the beginning of a technological process up to which the definite resource is to be engaged.

$$
\begin{gathered}
U^{(k+1)}=\left\{u_{1}^{(k+1)}, u_{2}^{(k+1)}, \ldots, u_{l-1}^{(k+1)}, u_{i}^{(k+1)}\right\}, \\
Z^{(k+1)}=\left[\begin{array}{llll}
z_{11}^{(k+1)} & z_{12}^{(k+1)} \ldots & z_{1 j}^{(k+1)} \ldots & z_{1 l}^{(k+1)} \\
z_{21}^{(k+1)} & z_{22}^{(k+1)} \ldots & z_{2 j}^{(k+1)} \ldots & z_{2 l}^{(k+1)} \\
z_{i 1}^{(k+1)} & z_{i 2}^{(k+1)} \ldots & z_{i j}^{(k+1)} \ldots & z_{i l}^{(k+1)} \\
z_{l 1}^{(k+1)} & z_{l 2}^{(k+1)} \ldots & z_{l j}^{(k+1)} \ldots & z_{m l}^{(k+1)}
\end{array}\right],
\end{gathered}
$$

where $u_{i}$ is the number of DMu resource for technological process marked by $i$-th symbol, $z_{i j}$ is the indication characterizing the possibility to use the $j$-th resource in the $i$-th technological process. It shows which user disposes of the DMu resource marked by $j$ symbol in the $i$-th process, $l$ is the number of technological processes, $n$ is the quantity of DMu resource.

After such a simplification, simulation of DMu resources distribution variants becomes simple. The algorithm for such simulation is presented in Fig 1.

The $1^{\text {st }}$ variant of DMu resources distribution is simulated from the process marked by the $1^{\text {st }}$ number and it is attributed by the DMu resource to the smallest number. The same principle is observed in relation to other processes and DMu resources are attributed successively, but it is checked whether the resource is engaged or not.

\section{Graphical method for determining DMu resources supplying degree of simulation system}

If we assume that technological processes foreseen in the complex technological process are technologically disconnected and each of the available DMu resources to be distributed may be technically used for performing a definite technological process, then the total possible number of DMu resources distribution variants will be:

$$
K^{\prime}=n^{l}
$$

where $n$ is total number of DMu resources to be distributed, $l$ is total number of technological processes (users) in the complex technological process.

In practice, such work conditions exist rarely. And more often one should take into consideration the technological links of separate processes. In the graph (Fig 2), the technological processes to be performed are presented. The number of available DMu resources and their suitability for a technological process are known. In order to have a possibility to calculate the number of variants for using DMu resources, a vertical line is drawn across the initial point of each job. Then we count how many times the line crosses other lines denoting jobs $\left(q_{i}\right)$.

1. The first variant of resource distribution is shaped according to the start of work successively from the first process and attributing to each process a free resource with the smallest number.

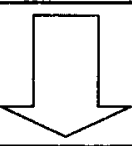

2. Shaping a new variant, we start with the last simulated variant process marked by the biggest number and satisfying the conditions of 3 and 4 items. The process marked by the biggest successive number is attributed in succession by an unapplied resource marked by a bigger number. To other following successive processes the free resources with smallest numbers are attributed successively.

3. If in the earlier variant the process with the biggest number was attributed by a biggest number or all the resources which could be attributed to the process with biggest number are used, we begin the redistribution with the process with the biggest number which is the nearest one to the process satisfying this condition and is attributed by a smaller number.

4. If the redistribution should start with the $1^{\text {st }}$ process, but in the earlier variant the $1^{\text {st }}$ process was attributed to the process with the biggest number, simulation of new variants is finished.

Fig 1. Algorithm for simulation of discretional resource supplying

On the base of these values, the degree of provision with DMu resources of the $i$-th user's (of the system to be simulated) is determined:

$$
k_{i}=n-q_{i}+1,
$$

where $n$ is total number of DMu resources to be distributed, $q_{i}$ - a count how many times the line crosses other lines denoting jobs. 
In physical sense, index $k_{i}$ expresses the available resource quantity which may be given to the $i$-th process.

If we assume that technological processes foreseen in the complex technological process are technologically disconnected and each of the available DMu resources to be distributed may be technically used for performing a definite technological process, the total number of the DMu resource distribution variants of the system to be simulated is determined according to the formula:

$$
K=\prod_{i=1}^{l} k_{i},
$$

where $l$ is total number of technological processes in which the distributed resource is to be used.

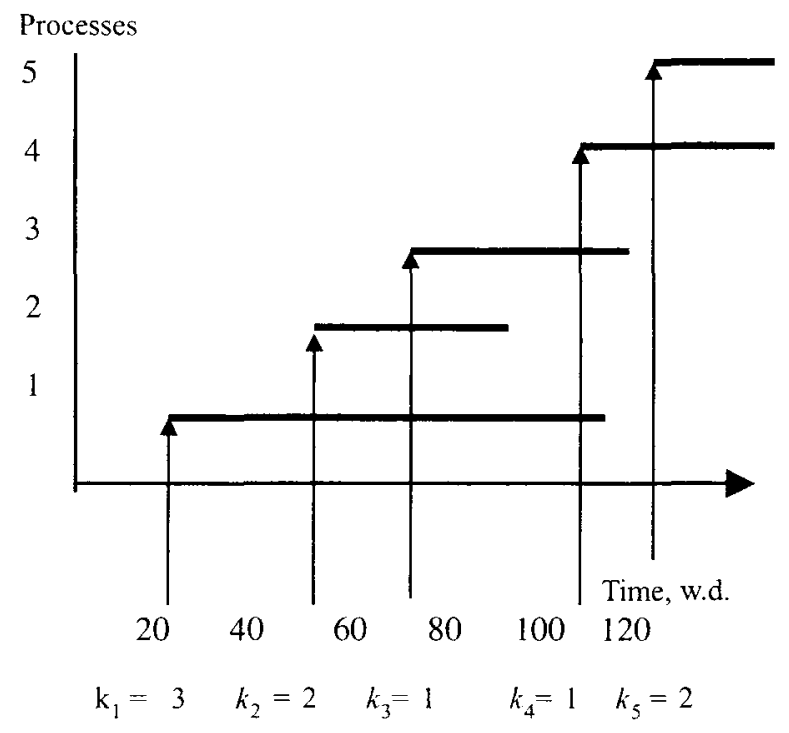

Fig 2. Schema for specifying discretional resource supplying degree of simulation system processes, when $X=6$

\section{The dependence of DMu resources distribution variants on the restriction degree of the system}

Depending on the character of technological process terms, the total number of DMu possible resource variants undergoes changes. To be more exact, the number of DMu resource distribution variants diminish when the total duration of work programme becomes shorter or when the degree of work programme conciseness increases; this restriction degree can be expressed by a summary power $X$.

$$
X=\sum_{i=1}^{l}\left(n-k_{i}\right) .
$$

The change of the total number of discrete resources distribution depending on the limitation degree of the system to be simulated can be expressed by empiric formulas:

$$
\left\{\begin{array}{l}
K_{\max }=\left(n-\frac{X}{l}\right) l \\
K_{\min }=n^{l}-X \cdot n^{l-l}, K_{\min }>0 .
\end{array}\right.
$$

Disregarding the system to be simulated in respect of time restrictions, for 6 processes the number of possible application variants of the available three $\mathrm{DMu}$ resources, the biggest number of DMu resources application variants would be:

$$
K^{\prime}=n^{l}=3^{6}=729 \text {. }
$$

The graphs (Fig 3) present the dependence of DMu resources distribution variants on the restriction degree of the system to be simulated and the number of DMu resources.

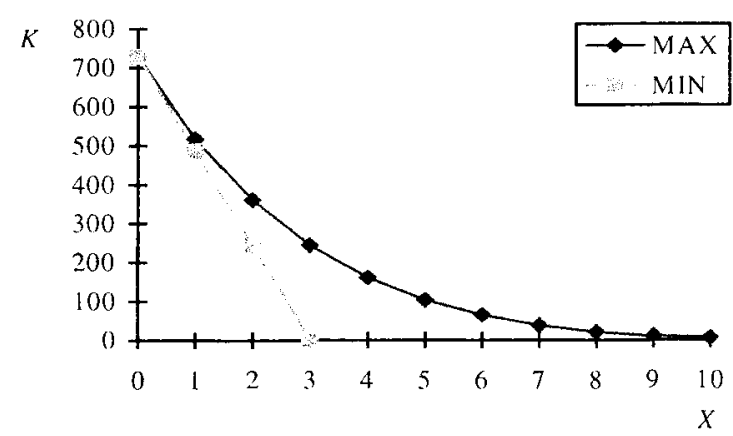

Fig 3. Relation between the number of variants $K$ discretional resource distribution and restriction degree $X$ of simulation system, when $n=3$ and $l=6$

\section{The influence of system restriction degree on the search for rational $D M u$ resource application vari- ants}

When decreasing the restriction degree of the system to be simulated, the number of DMu resource application variants increases and at the same time more favourable conditions for the search for rational variant develop.

Taking use of the schedule in Fig 2, let's determine for 5 processes the number of possible application variants of the available three DMu resources. Let's assume that each of the available distributing type DMu resources can be used for a definite technological process.

Disregarding the system to be simulated in respect of time restrictions, the biggest number of DMu resources application variants would be:

$$
K^{\prime}=n^{l}=3^{5}=243 .
$$

Taking into account the degree $X$ and disregarding the performance terms of definite processes of the system, the values of quantity $K$ may vary between $K_{\min }$ and $K_{\max }$.

$$
X=\sum_{i=1}^{l}\left(n-k_{i}\right)=0+1+2+2+1=6,
$$




$$
\begin{gathered}
k_{1}=n-q_{1}+1=3-1+1=3, \\
k_{2}=n-q_{2}+1=3-2+1=2, \\
k_{3}=n-q_{3}+1=3-3+1=1, \\
k_{4}=n-q_{4}+1=3-3+1=1, \\
k_{5}=n-q_{5}+1=3-2+1=2, \\
K_{\text {nin }}=n^{\prime}-X^{l-1} \cdot n=3^{5}-6^{4} \cdot 3<0, \\
\text { we accepl } \quad K_{\min }=0 . \\
K_{\text {max }}=\left(n-\frac{X}{l}\right)^{\prime}=\left(3-\frac{6}{5}\right)^{5}=19 .
\end{gathered}
$$

The graphs (Fig 4) present the relation between number of variants $K$ discretional resource distribution and restriction degree $X$ of simulation system, when $n=3$ and $l=5$.

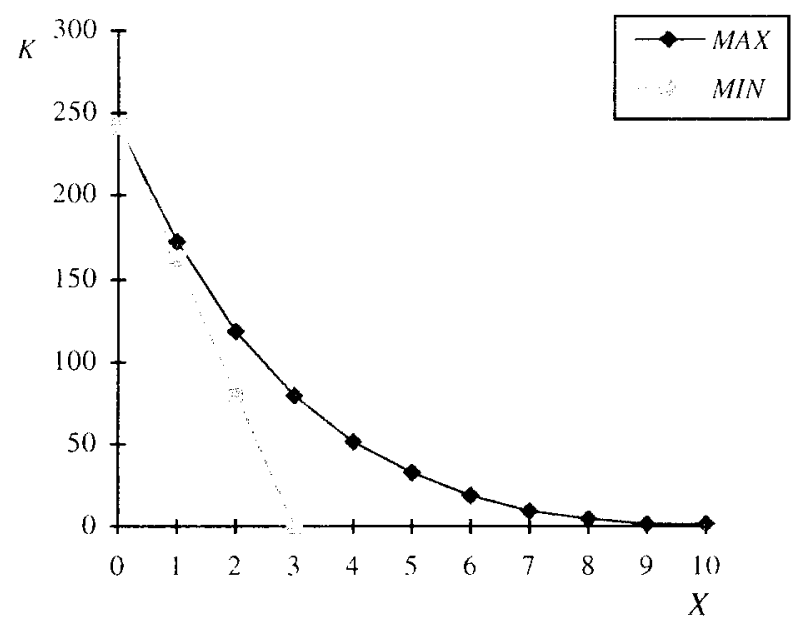

Fig 4. Schema for specifying discretional resource supplying degree of simulation system processes, when $l=5$, $n=3$

The real number of DMu resources application variants, taking into account definite process performance terms presented in Fig 2, will be:

$$
K=\prod_{i=1}^{l} k_{i}=3 \cdot 2 \cdot 1 \cdot 1 \cdot 2=12
$$

In this case, real restrictions of time have decreased by more than 20 times the possible number of DMu resources distribution variants, in comparison with the biggest possible number of variants. According to the algorithm for simulation of discretional resource supplying (Fig 1), taking use of the schedule in Fig 2, let's determine for 5 processes the possible application variants of the available three DMu resources.

$$
Z=\left[\begin{array}{lllll}
1 & 2 & 3 & 2 & 1 \\
1 & 2 & 3 & 2 & 3 \\
1 & 3 & 2 & 3 & 1 \\
1 & 3 & 2 & 3 & 2 \\
2 & 1 & 3 & 1 & 2 \\
2 & 1 & 3 & 1 & 3 \\
2 & 3 & 1 & 3 & 1 \\
2 & 3 & 1 & 3 & 2 \\
3 & 1 & 2 & 1 & 2 \\
3 & 1 & 2 & 1 & 3 \\
3 & 2 & 1 & 2 & 1 \\
3 & 2 & 1 & 2 & 3
\end{array}\right],
$$

The degree $X$ of system restriction having been decreased, the parameters of the problem discussed change entirely. By using data of Fig 2 and changing the work schedule of the second user's need for resources, as shown in Fig 5, the degree $X$ of the system's restriction is diminished:

$$
\begin{gathered}
k_{1}=n-q_{1}+1=3-1+1=3, \\
k_{2}=n-q_{2}+1=3-2+1=2, \\
k_{3}=n-q_{3}+1=3-2+1=2, \\
k_{4}=n-q_{4}+1=3-3+1=1, \\
k_{5}=n-q_{5}+1=3-2+1=2, \\
X=\sum_{i=1}^{l}\left(n-k_{i}\right)=0+1+1+2+1=5 .
\end{gathered}
$$

Processes

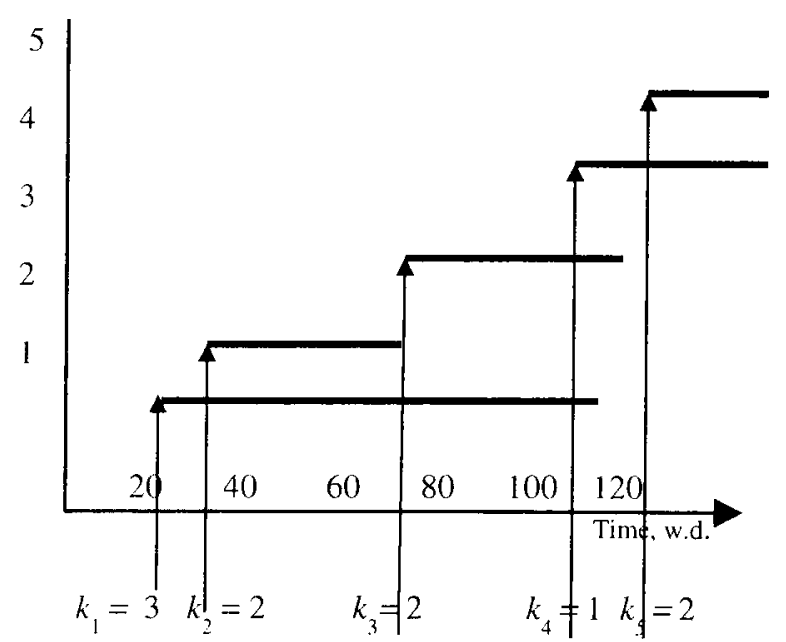

Fig 5. Schema for specifying discretional resource supplying degree of simulation system processes, when $X=5$ 
Taking into account the degree $X$ and disregarding the performance terms of definite processes of the system, the values of quantity $K$ may vary between $K_{m i n}$ and $K_{\max }$

$$
\begin{gathered}
K_{\text {min }}=n^{l}-X^{l-1} \cdot n=3^{5}-5^{4} \cdot 3<0, \\
\text { we accept } \quad K_{\text {min }}=0 . \\
K_{\text {max }}=\left(n-\frac{X}{l}\right)^{l}=\left(3-\frac{5}{5}\right)^{5}=32 .
\end{gathered}
$$

In this case, the real number of DMu resource application variants, taking into account definite terms of job performance given in Fig 5 , will be:

$$
\begin{aligned}
K=\prod_{i=1}^{l} k_{i}= & 3 \cdot 2 \cdot 2 \cdot 2 \cdot 1 \cdot 2 \\
Z & =\left[\begin{array}{lllll}
1 & 2 & 2 & 3 & 1 \\
1 & 2 & 2 & 3 & 2 \\
1 & 2 & 3 & 2 & 1 \\
1 & 2 & 3 & 2 & 3 \\
1 & 3 & 2 & 3 & 1 \\
1 & 3 & 2 & 3 & 2 \\
1 & 3 & 3 & 2 & 1 \\
1 & 3 & 3 & 2 & 3 \\
2 & 1 & 1 & 3 & 1 \\
2 & 1 & 1 & 3 & 2 \\
2 & 1 & 3 & 1 & 2 \\
2 & 1 & 3 & 1 & 3 \\
2 & 3 & 1 & 3 & 1 \\
2 & 3 & 1 & 3 & 2 \\
2 & 3 & 3 & 1 & 2 \\
2 & 3 & 3 & 1 & 3 \\
3 & 1 & 1 & 2 & 1 \\
3 & 1 & 1 & 2 & 3 \\
3 & 1 & 2 & 1 & 2 \\
3 & 1 & 2 & 1 & 3 \\
3 & 2 & 1 & 2 & 1 \\
3 & 2 & 1 & 2 & 3 \\
3 & 2 & 2 & 1 & 2 \\
3 & 2 & 2 & 1 & 3
\end{array}\right]
\end{aligned}
$$

Thus, when decreasing the total degree $X$ of system restriction, even without changing the duration of the system's realization, the possible number of DMu re- source application variants is increased considerably, and this results in widening the possibilities of search for a rational variant.

If we change terms of process No 3 (Fig 5) and start process No 3 ten days later, we will have absolutely different situation (Fig 6). The possible number of DMu resource application variants is decreased considerably.

Processes

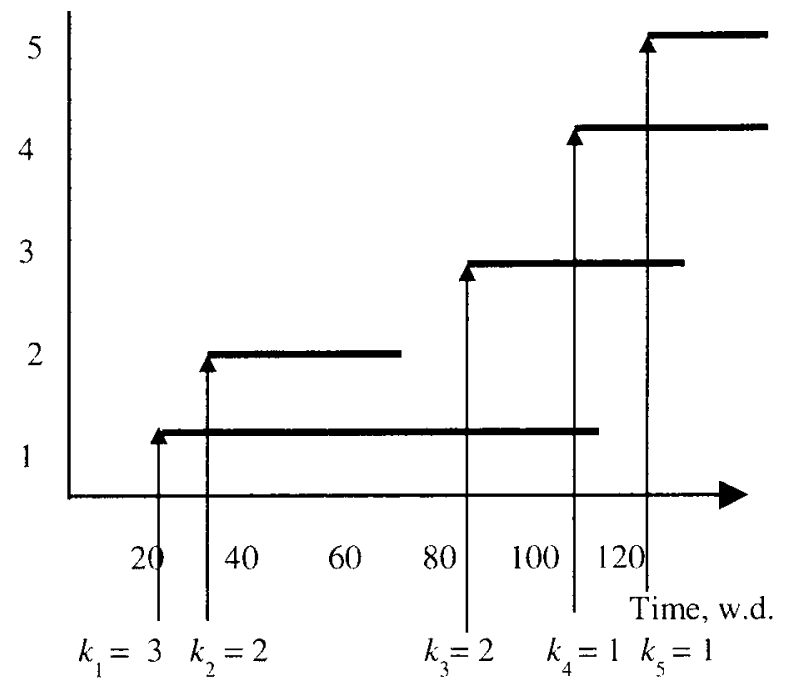

Fig 6. Schema for specifying discretional resource supplying degree of simulation system processes, when $X=6$

Depending on the character of technological process terms, the total degree of DMu resource restriction can be expressed by a summary power $X$.

$$
\begin{gathered}
X=\sum_{i=1}^{l}\left(n-k_{i}\right)=0+1+1+2+2=6 . \\
k_{1}=n-q_{1}+1=3-1+1=3, \\
k_{2}=n-q_{2}+1=3-2+1=2, \\
k_{3}=n-q_{3}+1=3-2+1=2, \\
k_{4}=n-q_{4}+1=3-3+1=1, \\
k_{5}=n-q_{5}+1=3-3+1=1 .
\end{gathered}
$$

Taking into account the degree $X$ and disregarding the performance terms of definite processes of the system, the values of quantity $\mathrm{K}$ may vary between $K_{m i n}$ and $K_{m a x}$ :

$$
\begin{gathered}
K_{\min }=n^{l}-X^{l-1} \cdot n=3^{5}-6^{4} \cdot 3<0, \\
\text { we accept } \quad K_{\text {min }}=0 . \\
K_{\text {max }}=\left(n-\frac{X}{l}\right)^{l}=\left(3-\frac{6}{5}\right)^{5}=19 .
\end{gathered}
$$


The real number of DMu resource application variants, taking into account definite process performance terms presented in Fig 6, will be:

$$
K=\prod_{i=1}^{l} k_{i}=3 \cdot 2 \cdot 2 \cdot 1 \cdot 1=12
$$

According to the algorithm for simulation of discretional resource supplying (Fig 1), taking use of the schedule in Fig 6, let's determine for 5 processes the possible application variants of the available three DMu resources:

$$
Z=\left[\begin{array}{lllll}
1 & 2 & 2 & 3 & 1 \\
1 & 2 & 3 & 2 & 1 \\
1 & 3 & 2 & 3 & 1 \\
1 & 3 & 3 & 2 & 1 \\
2 & 1 & 1 & 3 & 2 \\
2 & 1 & 3 & 1 & 2 \\
2 & 3 & 1 & 3 & 2 \\
2 & 3 & 3 & 1 & 2 \\
3 & 1 & 1 & 2 & 3 \\
3 & 1 & 2 & 1 & 3 \\
3 & 2 & 1 & 2 & 3 \\
3 & 2 & 2 & 1 & 3
\end{array}\right] .
$$

4. The dependence of DMu resource distribution variants on the restriction degree of the system when some resources do not satisfy some processes

Taking use of the schedule in Fig 7, let's determine for 4 processes the number of possible application variants of the available three DMu resources. Let's assume that each of the available distributing type of DMu resources can be used for a definite technological process.

Disregarding the system to be simulated in respect of time restrictions, the biggest number of DMu resource application variants would be:

$$
K^{\prime}=n^{l}=3^{4}=81 .
$$

Depending on the character of technological process terms in Fig 7, the total number of DMu resources restriction degree can be expressed by a summary power $X$.

$$
\begin{gathered}
X=\sum_{i=1}^{l}\left(n-k_{i}\right)=0+1+1+2=4 . \\
k_{1}=n-q_{1}+1=3-1+1=3, \\
k_{2}=n-q_{2}+1=3-2+1=2, \\
k_{3}=n-q_{3}+1=3-2+1=2, \\
k_{4}=n-q_{4}+1=3-3+1=1 .
\end{gathered}
$$

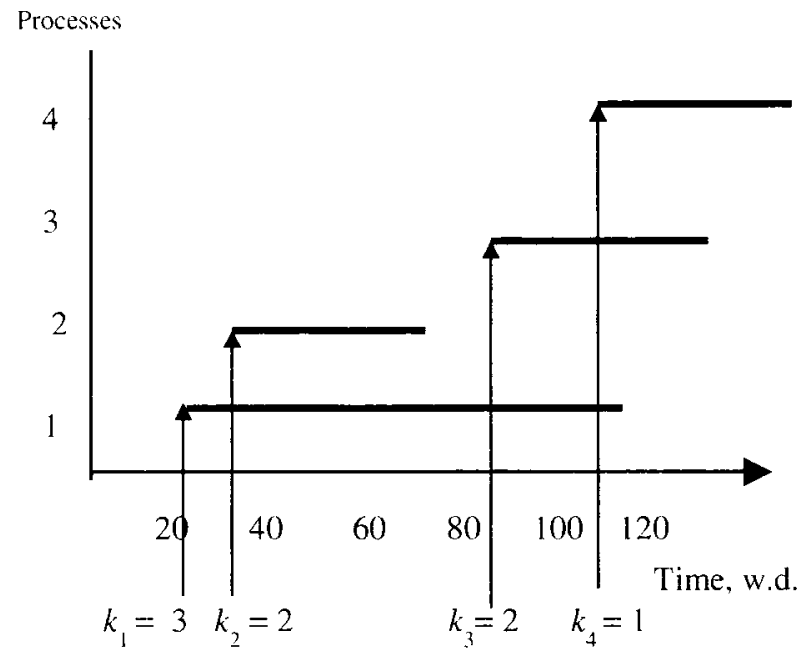

Fig 7. Scheme for specifying discretional resource supplying degree of simulated system processes, when $X=4$

Taking into account the degree $X$ and disregarding the performance terms of definite processes of the system, the values of quantity $K$ may vary between $K_{\text {min }}$ and $K_{\max }$ :

$$
\begin{gathered}
K_{\min }=n^{l}-X^{l-1} \cdot n=3^{4}-4^{3} \cdot 3<0, \\
\text { we accept } \quad K_{\text {min }}=0 . \\
K_{\text {max }}=\left(n-\frac{X}{l}\right)^{l}=\left(3-\frac{4}{4}\right)^{4}=16 .
\end{gathered}
$$

The real number of DMu resource application variants, taking into account definite process performance terms presented in Fig 7, will be:

$$
K=\prod_{i=1}^{l} k_{i}=3 \cdot 2 \cdot 2 \cdot 1=12
$$

According to the algorithm for simulation of discretional resources supplying (Fig 1), taking use of the schedule in Fig 7, let's determine for 4 processes the possible application variants of the available three DMu resources:

$$
Z=\left[\begin{array}{llll}
1 & 2 & 2 & 3 \\
1 & 2 & 3 & 2 \\
1 & 3 & 2 & 3 \\
1 & 3 & 3 & 2 \\
2 & 1 & 1 & 3 \\
2 & 1 & 3 & 1 \\
2 & 3 & 1 & 3 \\
2 & 3 & 3 & 1 \\
3 & 1 & 1 & 2 \\
3 & 1 & 2 & 1 \\
3 & 2 & 1 & 2 \\
3 & 2 & 2 & 1
\end{array}\right] .
$$


By using data of Fig 7 assume that the resource No 2 does not satisfy process No 1 and process No 4. On the base of these, according to the algorithm for simulation of discretional resource supplying (Fig 1), let's determine for 4 processes the possible application variants of the available Dmu:

$$
Z=\left[\begin{array}{llll}
1 & 2 & 2 & 3 \\
1 & 3 & 2 & 3 \\
3 & 1 & 2 & 1 \\
3 & 2 & 2 & 1
\end{array}\right] .
$$

The total amount of possible variants decreases $2 / 3$ times.

By using data of Fig 6 assume that resource No 2 does not satisfy process No 1 and process No 5. According to the algorithm for the simulation of discretional resources supplying (Fig 1), let's determine for 5 processes the possible application variants of the available DMu resources:

$$
Z=\left[\begin{array}{lllll}
1 & 2 & 2 & 3 & 1 \\
1 & 2 & 3 & 2 & 1 \\
1 & 3 & 2 & 3 & 1 \\
1 & 3 & 3 & 2 & 1 \\
3 & 1 & 1 & 2 & 3 \\
3 & 1 & 2 & 1 & 3 \\
3 & 2 & 1 & 2 & 3 \\
3 & 2 & 2 & 1 & 3
\end{array}\right]
$$

The total amount of possible variants decreases $1 / 3$ times.

As the experience shows, when resources do not satisfy processes, the largest decreasing of $D M u$ resource distribution variants can be $A / n$ times. $A$ - amount of restrictions when DMu resources can't be used for a definite technological processes. Minimal decrease of $\mathrm{DMu}$ resource distribution variants can be $1 / n$ times.

\section{Conclusions}

1. The article presents the dependence of DMu resource distribution variants on the degree of restriction of the system to be simulated.

2. As the experience shows, the largest amount of computation time may be saved and the problem solved in a most reasonable way, when the basic task parameters are calculated accurately during the first stage of simulation [10].

3. The degree of restriction of the system to be simulated is the one of basic parameters that must be determined during the first stage of simulation of DMu resource distribution variants.

\section{References}

1. Yeong, S.-C. The effect of human factors applied to simulation and resource optimization using computer simulation. Thesis. Iowa State University, 1999.

2. Afanasev, V. A. Algorithms for calculation and optimization methods of works management (Алгоритмы формирования расчета и оптимизашии методов организации раб̆т). Leningrad: Lenuprizdat, 1980 (in Russian).

3. Jeong, K.-C.; Kim, Y.-D. A real-time scheduling mechanism for a flexible manufacturing system: using simulation and dispatching rules. International Journal of Production Research, 36, No 9, 1998, p. 2609-2626.

4. Tamošaitis, R. Multipurpose estimation usefulness of cranes in erection works of multistory houses. PhD thesis (Многоцелевая оценка эффективности использования кранов при монтаже многоэтажных каркасных зданий. Автореферат диссертации на соискание ученой степени кандидата технических наук). Dnepropetrovsk Civil Engineering Institute, 1991 (in Russian).

5. Zavadskas, E. K.; Kaklauskas, A. A new Method of Complex Evaluation of Projects Based on Multiple Criteria Analysis and the Principle of Proportionality. In: $5^{\text {th }}$ International Conference "Integrating Fedinology and Human Decisions: Global Bridges into the 21 th Century". Decision Science Institute, 1999 Athens. Proceedings, Vol 2. Ed. by Dimitris K. Despotis, Constantin Zoporinidis, July 4-7, 1999, Athens, 1999, p. 2051-2053.

6. Zavadskas, E. K. Multiple criteria solutions in construction (Mehrkriterielle Entscheidungen in Bauwesen). Vilnius: Technika, 2000. 208 p. (in German).

7. Pritsker, A. Alan B.; Sigal, C. Elliott; Hammesfahr, R. D. Jack. Slam II network models for decision support [South San Francisco]: Scientific Press, 1994.

8. Pritsker, A. Alan B.; O'Reilly, Jean J.; LaVal, David K. Simulation with Visual SLAM and AweSim. New York: West Lafayette, Ind. : Wiley; Systems Pub. Corp., 1997.

9. Zavadskas, E. K.; Kaklauskas, A.; Lepkova, N.; Zalatorius, J. Facilities management multiple criteria analysis. Civil Engineering and Management (Statyba), Vol VII, No 6, 2001, p. 481-489 (in Lithuanian).

10. Tamošaitis, R. Simulation of Technological Processes of Civil Engineering Companies. Informatica, Vol 12, No 4, 2001, p. 585-592. 